\title{
A Study of the Impact of Chinese Star Cai Xukun Starring in the Prada Commercial Code Human on Chinese Young Consumers
}

\author{
Ji Shenran ${ }^{1 *}$ \\ ${ }^{1}$ Ji Shenran, Keystone Academy, Beijing, China \\ *Ji Shenran, Email: jiayi.zhu@gecacademy.cn
}

\begin{abstract}
Prada is an Italian luxury brand founded in 1913 that offers high-quality leather goods, clothing, and footwear for men and women. On May 31, 2019, Prada's official Weibo announced that the famous Chinese singer Cai Xukun became its Chinese spokesperson. Cai Xukun is a singer and actor who is popular and loved by many young people in China. At the same time, his identity is controversial, as many people doubt his performance style and skills. Code Human is a commercial campaign released by Prada on June 2, 2019, starring Cai Xukun to showcase Prada's Fall/Winter 2019 menswear collection. As of July 15, 2021, Code Human has received 555,000 retweets, 59,000 comments, and 401,000 likes on Weibo. This paper will investigate the impact of the Prada commercial Code Human starring Chinese star Cai Xukun on Chinese young consumers. The content helps to understand Chinese consumers' consumption psychology and motivation and provides a strong reference for the development of other luxury industries in the Chinese market. The survey was used to investigate the topic and was sent out on Weibo, a popular social networking application used by young people in China. Based on the results collected from 107 valid questionaries, Cai Xukun's controversial image in the public eye led to a polarization in the questionnaire answers. Meanwhile, the clothes worn by Cai Xukun in the commercial were the main reason why consumers did not arouse their desire to buy Prada products, so Prada needed to change its design to cater to the public's aesthetics. The analysis shows that the image of Xukun Cai is not suitable to be the spokesperson of Prada. Therefore, it is recommended that Prada change its brand spokesperson in order to gain more attention and favor from younger consumers.
\end{abstract}

Keywords: Prada, Cai Xukun, Code Human, Chinese young consumers

\section{INTRODUCTION}

Prada, an Italian luxury brand, is best known for its high-quality leather goods [1]. The brand was established in 1913 by Mario Prada, who opened the first store in Milan. It was not until the late 1970s that Mario Prada's granddaughter Miuccia Prada took over the brand. With Patrizio Bertelli who has extensive experience in the production of luxury products, they lay the foundation for the future international expansion of Prada. Today, Prada provides leather goods, clothing, and footwear for men and women, combining modern, innovative and refined design with unique handmade items. Prada has also branched out into eyewear and perfume [2]. In order to have better competitive power among other luxury brands, Prada has use celebrity endorsements as one of their marketing strategies. On
May 31, 2019, Prada's official Weibo account announced Chinese famous singer Cai Xukun to become its youngest Chinese brand spokesperson [3].

Cai Xukun was born on August 2, 1998. He is a singer, music producer, MV director, and actor. In 2018, participating in a Chinese talent show called Idol Producer brought him a lot of fame, however this fame is controversial due to his performance style and skills. Cai Xukun's popularity grew and he continues making progress in his field. He has released his songs such as "One," "Home," and "Lover," which are successful and appreciable. By 2020, he won several honors, including first place in the Tencent Entertainment White Paper on the commercial value of male stars 2020 and the most influential singing-songwriter in the TMEA Tencent Music Entertainment Ceremony [4]. 
Code Human is a commercial advertisement film released on June 2, 2019, by Prada, starring Cai Xukun for showcasing the Prada Fall/Winter 2019 menswear collection. Prada collaborated with Chinese artist Cao Fei to create this project. According to $\mathrm{Cao} F \mathrm{Fei}$, the notion of the Code Human originates from Aidoru (Japanese pronunciation of English word "idol"), the image packaging and marketing of contemporary pop culture stars. As now the Idol culture is a feeding mechanism based on the interaction between fans and idols on the Internet platform, the idea of Code Human is to question people: Is perfection the ultimate truth about a figure so admired and so desired by fans? Or is it an unattainable delusion? In the film, the setting is a "Museum of Future Human," where the perfect artificial human Cai Xukun stands motionless in the specimen cabinet of the exhibition area of "Post-Anthropocene" for the viewers to appreciate. Cai Xukun also plays a visitor role in the museum. He suddenly sees a specimen that looks almost exactly like himself in the exhibition hall. Is the specimen imitating him, or is he just a copy of the specimen? It may be that the ethics of technology that artificial humans will face in the future world, like cloning, will eventually lead to a hedge between species [5]. Until July 15, 2021, Code Human has received 555,000 retweets, 59,000 comments, and 401,000 likes on Weibo. Cai Xukun's fans left comments at the bottom of the video, posting photos of the Prada products they bought, with the words "I love the design philosophy of Prada, and will continue to support Prada and Prada's spokesperson Cai Xukun."

Many literatures focus on the relationship between advertising and consumers, which can be categorized into two perspectives: macro perspective and micro perspective.

From a macro perspective, literature discussed the overall impact of celebrity endorsement on consumers. In 2018, the paper "Influence of celebrity endorsement on consumer purchasing behaviours" written by Quan Qingying, Liang Qiang, and Sun Yan was published on "Liaoning Economy". The article mentioned that the authenticity of celebrity endorsement advertisements has a great impact on consumers' purchasing behaviours. Once negative news is derived from the advertisements, the image of the products and celebrities will be damaged. In addition, a celebrity's image affects the products he/she endorses [6].

"A study on the effectiveness of purchasing behaviours in consumer market and celebrity endorsement advertisements" by $\mathrm{Hu}$ Shitao was published in "China Water Transport" in 2009. In the study, the author finds that when consumers buy goods with fashion nature, such as high-end clothing, cosmetics, and mobile phones, celebrity endorsement is of great significance. For daily necessities such as vinegar, salt, toothbrush, and toothpaste, celebrity endorsement has some effects for consumers [7]. The article "A brief discussion on celebrity endorsement and its influence on brand loyalty" published on "Modern Business" was written by Li Jingjing in 2016. The author wrote the more credibility a star is, the more likely consumers are to have positive comments on the products or services he/she endorses, thus promoting the brand based on the consumption of fans [8].

From a micro perspective, literature focused on the impact of specific celebrity endorsements in a particular business field on consumers. "Study on Star Endorsement Strategy of Domestic Smart Phone" was written by Guo Xiaomeng in 2019. The study reveals although there are doubts about celebrity endorsement in the industry at present, saying it is too expensive and is better to spend the money on product research and development. By looking at the current domestic smartphone market, celebrity endorsement is practical [9].

The paper "Influence of makeup spokesperson on consumer brand attitude" by Wen Hongyan was published in 2019. The author did research and founds out that the celebrity endorsement of beauty products with high appearance level is significantly better than the celebrity endorsement with low appearance level in terms of consumers' behavioural effect and consumers' brand cognition effect [10]. In 2019, "Study on the Influence of Print Advertisements of Professional Sports Products Endorsed by Sports Stars on Consumers' Purchasing Decisions: A Case Study of Printed Advertisements of Tennis Rackets" written by Lv Xingyang, Guoxuan, and Liu Yue was published in "Journal of Capital University of Physical Education and Sports". Based on the article, the influence of sports stars' endorsement of print ads on the consumers' purchasing decisions was not significant, while the sales model and purchasing experience had an influence on the consumers' purchasing decisions [11].

All the above pieces of literatures either discuss the overall impact of celebrity endorsements on consumers, or focus on individual markets, such as sports, makeup, mobile phones. No study has an in-depth understanding of top stars' endorsement of supreme luxury. This research gap must be filled, which will have a better impact on China's marketing development. Although this paper focuses on the advertisement of Cai Xukun's participation in Prada's Code Human, its content can also provide a powerful reference for the development of other luxury industries in the Chinese market. This paper could help in understanding the consumption mentality and consumption motivation of Chinese consumers. This paper takes the Prada advertisement Code Human performed by Cai Xukun as an example, using the method of survey to study the impact of this advertisement on Chinese young consumers. 


\section{METHODOLOGY}

The survey will be used to investigate the influence of Prada's advertisement Code Human starring Cai Xukun, on Chinese young consumers. According to the "Dictionary of Australian Education," survey is a method of collecting data in the form of a questionnaire for research or other purposes. Data includes factual information and opinions. Questionnaires are widely used in educational research and have a general nature. The data from the questionnaires are often fed into a computer for analysis, not only to analyze the opinions but also to examine other connections between the data to explain the differences in opinions [12]. Therefore, the survey is used in this paper to understand the opinions that young Chinese consumers had on Cai Xukun in the Prada advertisement. The results from the survey would help to know the degree of success of Cai Xukun being the spokesperson of Prada from consumers' points of view.

The questionnaire was sent out on Weibo, a popular social networking app used by young people in China. The questionnaire was handed out for a week. A total of 114 questionnaires were collected and 107 were valid. The identification criteria for invalid questionnaires are: same IP address, identical answers in every questionnaire, or the responses show apparent regularity. Based on the statistics, $38.6 \%$ of the respondents were male and $61.4 \%$ were female. They were mainly young between 18 and 25 years old, which occupied $75.4 \%$.

In the process of questionnaire delivery, due to lack of financial support, no cash or gifts were given to respondents, which may have some impacts on the accuracy of the questionnaire. All respondents will appear anonymous and their answers will be kept strictly confidential.

\section{RESULTS AND DISCUSSION}

This paper aims to understand how Cai Xukun's endorsement of Prada's Code Human advertisement influences young Chinese consumers.

According to the survey, $66.7 \%$ of respondents claimed they either "prefer" or "very prefer" the image of Cai Xukun in the advertisement, with $19.3 \%$ respondents who felt "just so so", and $14.0 \%$ respondents either "dislike" or "very dislike". This result suggests that most people favor the image of Cai Xukun in Code Human. For those respondents who chose "prefer" or "very prefer", there were $69.3 \%$ of them said the gorgeous face of Cai Xukun attracted them in the advertisement, and $52.6 \%$ chose costume, $50.9 \%$ chose hairstyle. Since Cai Xukun wears Prada clothes in the advertisement, more than $50 \%$ of people notice and like these clothes, which indicates that the advertisement has a certain effect on consumers. Besides this, it can also be seen from the results that Cai Xukun's handsome face and hairstyle account for a large proportion of people's liking. Therefore, when making a luxury advertisement, producers should pay attention to the appearance level and hairstyle of the advertiser. Especially appearance is essential in such luxury brands, so producers should prioritize those celebrities with good-looking faces. Although some celebrities may have excellent acting skills, high popularity, and honor, they are not suitable for luxury advertising if they lack delicate and beautiful faces.

$43.9 \%$ of the respondents said that the image of Cai Xukun in the Code Human kindled their desire to buy Prada products, while $39.5 \%$ of the respondents did not. The rest of the respondents $(16.7 \%)$ said they had no feelings for Cai Xukun. Looking at these results, the proportion of the two choices between willing to buy and not willing to buy is very close. In other words, the results suggest there is polarization. This polarizing phenomenon may be due to the public impression of Cai Xukun. Despite his popularity in China, Cai Xukun's image is very polarizing. His fans are very supportive of him, while those who are not his fans hate him. This stems mainly from an incident that happened in 2019. Cai Xukun once performed a controversial video of himself playing basketball on a talent show, with many viewers commenting on his skills. The basketball video was then edited into a funny video and posted on the Internet. Before this, many stars' videos were uploaded as funny videos on the Internet, and they never took any action. Those kinds of videos often amused netizens. But Cai Xukun did send a lawyer's letter to the website that uploaded his hilarious video, asking it to take the video down. This behavior made many netizens feel that he has no entertainment spirit. It is a big reason why many people dislike him. The results suggest Prada's advertising still has a lot of room for improvement. For those respondents who did not think Cai Xukun kindled their desire to buy Prada products, $42.2 \%$ of them said it was because they did not like his clothes in the advertisement. $26.7 \%$ of respondents did not like his face, $20 \%$ did not like his hairstyle, and $6.7 \%$ did not like him as a person. It can be seen that the dislike of Cai Xukun's clothes in the advertisement is the main reason why respondents did not have the desire to buy Prada products. Interestingly, so many consumers do not like the clothes Cai Xukun wears in the ads, which he is endorsing. This suggests a gap between the brand's design and the consumers' aesthetic. Prada may need to change its design to cater to consumers' tastes.

$48.3 \%$ of respondents said it would be better if Code Human switched to other stars, $36.0 \%$ said it would be worse, and $15.8 \%$ said it would not make any difference. This indicates that celebrity endorsement of luxury products does have a great impact on consumers. Most people have subjective consciousness and will 
have different feelings on different celebrity endorsements. Furthermore, $62.3 \%$ of the respondents think that Cai Xukun, as the spokesperson, does not show the brand image of Prada. These results suggest Cai Xunkun is not the best person for the advertisement. Maybe Prada should consider changing its advertising spokesman to persuade consumers to purchase Prada products and get more attention for the brand.

According to the survey data, $66.7 \%$ of respondents liked his image in the advertisements, but $62.3 \%$ thought that he did not show the image of Prada. This indicates that Cai Xukun is not suitable for the adverting of Prada.

\section{CONCLUSION}

The impact of Chinese star Cai Xukun's advertisement for Prada's Code Human on young Chinese consumers has been investigated. In general, Cai Xukun's self-image in the public's eyes has led to the polarization of results. Half liked his overall image in the advertisement, and the other half did not. At the same time, the clothes Cai Xukun wears in the ad are the main reason consumers do not arouse their desire to buy Prada products, so Prada needs to change its design to cater to the public aesthetic. Analysis shows that Cai Xukun is not suitable for Prada's spokesperson due to his unsuitable and controversial image. Therefore, it is suggested that Prada should change its brand spokesperson so as to gain more attention and favor from young consumers.

\section{REFERENCES}

[1] Fashion Chinese Brand Library, Prada Story, Retrieved from http://brand.youngchina.cn/prada/introduce/

[2] Prada Group, Company Profile, Retrieved from https://www.pradagroup.com/content/dam/pradagr oup/documents/Group_Profile/Company\%20profil e\%20Prada\%20Group_ENG.pdf
[3] Jing Daily, Chinese Whispers: Prada Names a Gen$\mathrm{Z}$ Spokesperson to Rejuvenate the Brand, and More, Retrieved from https://jingdaily.com/pradachinese-spokesperson/

[4] Photo home, CAI Xukun profile, Retrieved from https://www.tupianzj.com/ziliao/20180315/157209. html

[5] PRADA, Human Code, Retrieved from https://www.prada.com/cn/zh/pradasphere/specialprojects/2019/code-human.html

[6] Quan Qingying, LIANG Qiang, SUN Yan. The Impact of Celebrity endorsement on consumer purchasing Behavior [J]. Liaoning Economy,2018(08):74-76.

[7] $\mathrm{Hu}$ Shitao. Discussion on the effectiveness of consumer market purchasing behavior and celebrity endorsement advertising [J]. China water transport,2009,9(10):71-72.

[8] Li Jingjing. On celebrity Endorsement and its Influence on Brand Loyalty [J]. Modern Business, 2016(04):98-99.

[9] Guo Xiaomeng. Research on Celebrity Endorsement Strategy of Domestic smartphone [D]. Heilongjiang University, 2019.

[10] Wen Hongyan. The Influence of beauty makeup reverse spokesperson on Consumers' Brand Attitude [D]. Jinan University, 2019.

[11] Lu Xing-yang, GUO Xuan, Liu Yue. A study on the impact of professional sports Goods print ads endorsed by Sports Stars on consumers' purchasing decisions: A case study of tennis racket print Ads [J]. Journal of capital institute of physical education, 2019, 31(03):215-220.

[12] Wang Guofu, Wang Xiuling. Australian Educational Dictionary. Wuhan: Wuhan University Press. 2002. 